\title{
Influenza-infected newborn and adult monkeys exhibit a strong primary antibody response to hemagglutinin stem
}

\author{
Elene Clemens, ${ }^{1}$ Davide Angeletti, ${ }^{2}$ Beth C. Holbrook, ${ }^{1}$ Masaru Kanekiyo, ${ }^{4}$ Matthew J. Jorgensen, ${ }^{3}$ \\ Barney S. Graham, ${ }^{4}$ Jonathan Yewdell, ${ }^{5}$ and Martha A. Alexander-Miller ${ }^{1}$ \\ 'Department of Microbiology and Immunology, Wake Forest School of Medicine, Winston-Salem, North Carolina, USA. \\ 2Department of Microbiology and Immunology, Institute of Biomedicine, University of Gothenburg, Göteborg, Sweden. \\ ${ }^{3}$ Section on Comparative Medicine, Department of Pathology, Wake Forest School of Medicine, Winston-Salem, North \\ Carolina, USA. ${ }^{4}$ Vaccine Research Center and ${ }^{5}$ Laboratory of Viral Diseases, National Institute of Allergy and Infectious \\ Diseases, NIH, Bethesda, Maryland, USA.
}

\begin{abstract}
The specificity of antibodies (Abs) generated against influenza A virus (IAV) infection can significantly alter protection and viral clearance. At present, the impact of age upon this process is relatively unexplored. Here, we evaluated the $A b$ response in newborn and adult African green monkeys following infection with IAV using a strain that enables us to determine the immunodominance (ID) hierarchy of the Ab response to hemagglutinin (HA), the principal target of protective Abs. This revealed altered ID patterns in the early IgM anti-HA response in newborns versus adults that converged over time. While the IgC ID profiles for HA in newborn and adult monkeys were similar, this was not the case for IgA. Importantly, HA stem-specific Abs were generated robustly and similarly in newborns and adults in terms of quality and quantity. Together, these results demonstrate that newborns and adults can differ in the Ab ID pattern established following infection and that the ID pattern can vary across isotypes. In addition, newborns have the ability to generate potent HA stem-specific Ab responses. Our findings further the understanding of the newborn response to IAV antigens and inform the development of improved vaccines for this at-risk population.
\end{abstract}

Conflict of interest: The authors have declared that no conflict of interest exists.

Copyright: () 2020, American Society for Clinical Investigation.

Submitted: December 3, 2019

Accepted: February 12, 2020

Published: February 20, 2020

Reference information: /CI Insight. 2020;5(5):e135449.

https://doi.org/10.1172/jici.

insight.135449.

\section{Introduction}

At birth, infants transition from the protected environment of the womb to the myriad dangers of the outside world. This requires substantial adjustment from the neonatal immune system as it encounters its first antigenic challenges. At early times after birth, the immune system exists in an altered state that has been proposed to provide evolutionary benefit by allowing colonization of commensal microbiota; however, it leaves neonates more susceptible to infection (1,2). Influenza A virus (IAV) is a common respiratory tract infection that, compared with infection in older individuals, exhibits higher attack rates and higher incidences of serious disease and secondary complications in infants (3-5). This increased susceptibility, coupled with the lack of an approved influenza vaccine for infants under 6 months of age, leaves neonates vulnerable to IAV infection (6).

Ineffective neonatal immunity to IAV likely results from multiple defects in innate and adaptive mechanisms (7-9). These include diminished antibody $(\mathrm{Ab})$ responses, which could reflect deficiencies in B cell activation, somatic hypermutation, and class switching (10-14). A nearly completely unknown factor is the specificity of neonatal Abs relative to those found in older individuals. IAV has a number of immunogenic proteins, the most important of which for vaccination purposes is the hemagglutinin (HA) molecule, the target of the most potent neutralizing Abs in vitro and protective Abs in vivo.

HA is a homotrimeric glycoprotein with a highly variable globular domain sitting atop a more conserved stalk. The variability of the globular domain accounts for IAV's rapid antigenic drift and necessity for frequent vaccine updates. Previous studies have demonstrated that the H1 HA, as exemplified by the prototypical A/Puerto Rico/8/34 (H1N1) (PR8) strain, has 5 major antigenic sites in the globular domain recognized by Abs that neutralize viral infectivity (15). Recognition of these 5 sites can inform $\mathrm{Ab}$ function; 
for example, Abs binding to the Sa and Sb sites near the receptor binding site have been shown to be particularly efficacious in neutralizing viral entry, while Abs against other sites may be higher affinity or inhibit other steps of the viral life cycle. Recently, Ab against Sa was also shown to neutralize the 2009 pandemic strain of H1N1, suggesting that different head epitopes may be more or less susceptible to antigenic drift (16). Using the PR8 strain, Angeletti et al. described a system that enables quantitation of both Abs and B cells specific for each of the 5 sites (17). Applying this to the mouse model, they reported that Ab immunodominance (ID) hierarchy is consistent within a given mouse strain, evolves over time after immunization, and is altered by the nature of the immunizing event (i.e., vaccination vs. infection). In mice, as in multiple animal models and humans, the globular domain is highly immunodominant over the stem, which in naive animals exposed to either infection or vaccination elicits a minor $\mathrm{Ab}$ response.

As with T cell ID (18), multiple mechanisms likely contribute to Ab ID, the details of which will take a major effort to unravel. With the urgent need to improve IAV vaccines (which under the best circumstances provide protection in only $\sim 60 \%$ of recipients), it is critical to better understand Ab ID and how it affects protection, given that $\mathrm{Ab}$ specificity and heavy chain class govern the capacity for protection and crossstrain reactivity (19-22).

Virtually nothing is known about age-related alterations in Ab ID. This is an important topic given the high susceptibility of neonates to IAV and the potential long-term effects of childhood vaccination on lifelong IAV immunity, as evidence suggests that first exposure to IAV antigens can mold the lifetime response (23). Here, we investigate HA-specific Ab ID in newborn and adult African green monkeys (AGMs) following IAV infection, a model chosen because of the similarities between nonhuman primate (NHP) and human infant immune system development and function (24).

\section{Results}

Newborns and adults generate high levels of HA-specific Abs in response to IAV infection. To characterize age-related differences in HA Ab ID, we infected AGM newborns (6-10 days of age) or adults (6-9 years of age) with influenza virus strain PR8 via a combination of intranasal and intratracheal routes, measuring Ab responses at day 8 and day 14 after infection (p.i.). For preimmune samples, we collected adult blood at the time of infection; for newborns, we used samples from an age-matched naive cohort. As expected, HA-specific IgG and IgA Abs were not detectable by ELISA using purified HA as antigen in samples collected prior to infection, confirming that all infants and adults were initially influenza naive (Figure 1). HA-specific IgM was not detected in neonates but was present at low levels in naive adults (Figure 1A). At either day 8 or day 14 p.i., the amount of plasma HA-specific IgM, IgG, or IgA Ab was similar in newborns and adults (Figure 1), consistent with our previous analysis of total IAV-specific IgG and IgM Ab responses (9). As expected, levels of HA-specific IgG and IgA in bronchoalveolar lavage (BAL) corresponded to plasma titers (Figure 1, B and C). However, the concordance between total virus-specific and HA-specific Ab was not as clear for IgA. Although there was a trend toward higher anti-HA IgA Ab in adults (Figure 1), this did not reach statistical significance, as it did when total virus-specific IgA was measured in our previous studies (9). This finding suggests the fraction of Abs specific for individual viral proteins differs across Ab isotypes.

The IgM ID profile differs in adults versus newborns. In a variety of species ranging from lampreys to birds to mammals, nearly all Abs that bind the globular head domain of HA recognize amino acids present in 1 or more of the 5 major antigenic sites $(15,25)$. To measure specific $\mathrm{Ab}$ responses to these sites, we performed ELISAs using purified HA from a $4 \Delta \mathrm{HA}$ panel of 5 viruses that were generated by multiple rounds of selection with neutralizing mAbs to lose antigenicity in 4 of 5 antigenic sites (17). In addition, we used WT virus and S12, a strain that has lost antigenicity at all 5 sites because of 12 rounds of sequential selection with different neutralizing mAbs (26). The AUC for binding to each $4 \Delta \mathrm{HA}$ construct was normalized to the AUC for the WT HA molecule to allow comparison of ID hierarchy between animals.

The IgM response in newborns at day 8 p.i. exhibited a distinct ID hierarchy, with $\mathrm{Cb}$ and $\mathrm{Sb}$ being dominant (Figure 2A). By day 14 p.i. in neonates, Sb was solely dominant, with responses to the other 4 sites being nearly equal in magnitude (Figure $2 \mathrm{~B}$ ). Interestingly, in adults none of the sites demonstrated ID at either time point (Figure 2). These findings are consistent with the conclusion that the ID hierarchy for IgM anti-HA Abs differs between newborns and adults.

Plasma IgG and IgA anti-HA Ab responses form clear ID hierarchies at day 14 p.i. in both newborns and adults. We then assessed the ID hierarchy of IgG anti-HA at day 14 p.i. IgG ID hierarchy was not assessed at day 8 p.i. because IgG Abs were not reliably detectable this early after infection, likely because of the time 
A
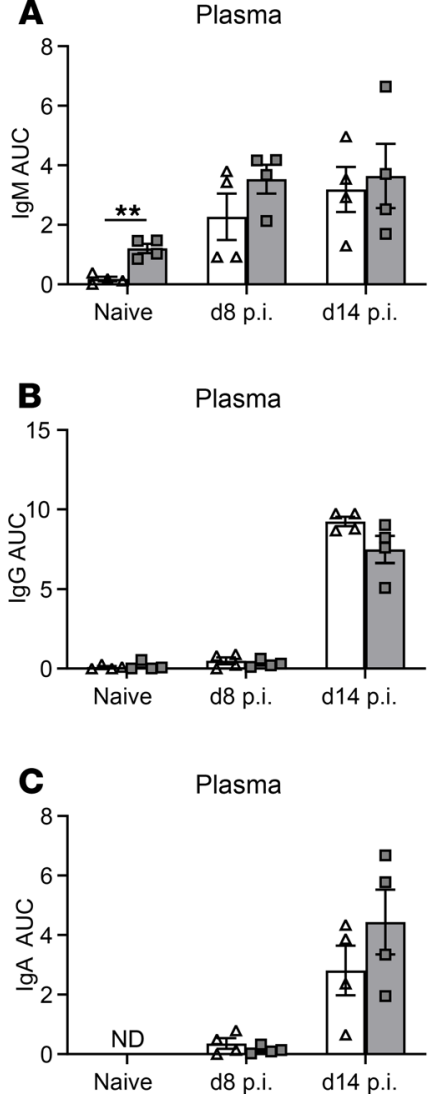
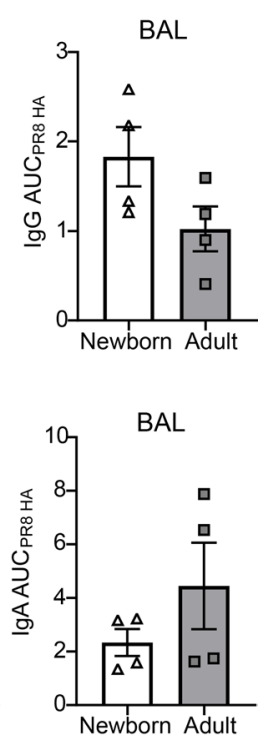

Figure 1. Ab responses to HA in newborn and adult AGMs following IAV infection. Newborn and adult AGMs ( $n=4$ /group) were infected with PR8 by the combined intranasal and intratracheal routes. Following infection, blood was drawn on days 8 and 14 . To assess naive responses, blood was collected from the adult animals at the time of infection and from age-matched controls for the newborn samples. IgM (A), IgG (B), and IgA (C) Abs against PR8 HA were measured by ELISA using plates coated with $5 \mathrm{ng}$ of PR8 HA. Plasma (left) or bronchoalveolar lavage fluid (right) collected at day 14 p.i. was added starting at $1: 10$ and $1: 5$, respectively, with subsequent 2 -fold dilutions. Total HA binding was quantified by calculating the area under the curve (AUC) for each animal. $\mathrm{HA}$-specific IgA was not detected (ND) in plasma from naive newborn or adult AGMs. Significance was determined by unpaired 1-tailed $t$ test; ${ }^{* *} P<0.01$.

required to produce high amounts of class-switched Abs. IgG Abs against $\mathrm{Cb}$ were clearly immunodominant in both newborns and adults (Figure 3A). For IgA Abs, by day 14 p.i., both newborns and adults responded to all 5 sites based on their values relative to S12 (Figure $3 \mathrm{~B}$ ). In newborns Sb was dominant, with $\mathrm{Ca} 1$ and $\mathrm{Cb}$ subdominant, while in adults $\mathrm{Sb}$ and $\mathrm{Cb}$ were codominant (Figure $3 \mathrm{~B}$ ). The hierarchy patterns were consistent for all individuals within each age group and isotype.

The ID hierarchy of HA-specific IgG Abs does not correlate with Ab avidity. We next examined the correlation between IgG Ab avidity for a given site with its position in the ID hierarchy at day 14 p.i. To estimate Ab avidity, we measured the amount of the chaotropic reagent sodium thiocyanate $(\mathrm{NaSCN})$ required to decrease $\mathrm{Ab}$ binding by $50 \%\left(\mathrm{IC}_{50}\right)$ in ELISAs using purified HA. Though clearly an imperfect measure of Ab avidity, which is difficult to measure in polyclonal Abs, many studies have found a good correlation between binding avidity and $\mathrm{Ab}$ avidity (27), and for HA in particular, a direct correlation between resistance to chaotropic agents and $\mathrm{Ab}$ off-rates (28).

No correlation between the $\mathrm{IC}_{50}$ for IgG binding and ID was present; i.e., the avidity of the immunodominant $\mathrm{Cb}$-specific $\mathrm{Ab}$ was not consistently either higher or lower than other epitopes (Figure 4). Ca2 was the only site in adults to which IgG Abs had a significantly lower avidity; however, this difference was not reflected in the ID hierarchy (Figure 4 vs. Figure 2). Interestingly, Abs against the dominant $\mathrm{Cb}$ epitope generated by newborns had a significantly lower avidity compared with those from adults $(0.67$ vs. $0.79, P=0.028)$. This difference was restricted to the $\mathrm{Cb}$-specific Abs, and it was not observed for Abs against the other epitopes.

NHPs generate a robust $H A$ stem-specific Ab response. In addition to Abs directed against epitopes in the HA head region, individuals also produce Abs against the more highly conserved stem region of the HA molecule (29-32). In humans, these Abs appear to be subdominant, as they are represented at significantly lower levels than those directed against the head region $(31,32)$. The robust response against S12, which lacks the frequently recognized head epitopes, that was observed in both adult and newborn animals suggested that NHPs may generate responses to the HA stem region. Alternatively, the animals could be producing Abs against alternative epitopes present in the head portion of HA or against contaminating virus proteins in our preparation. 
A

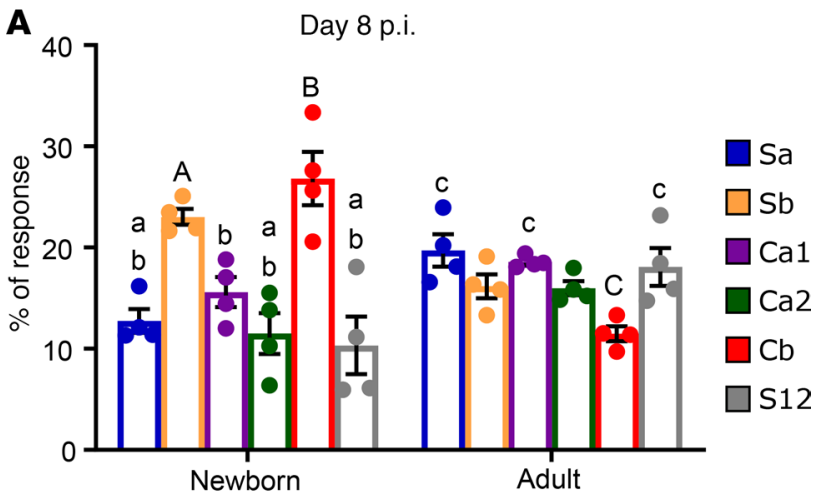

B

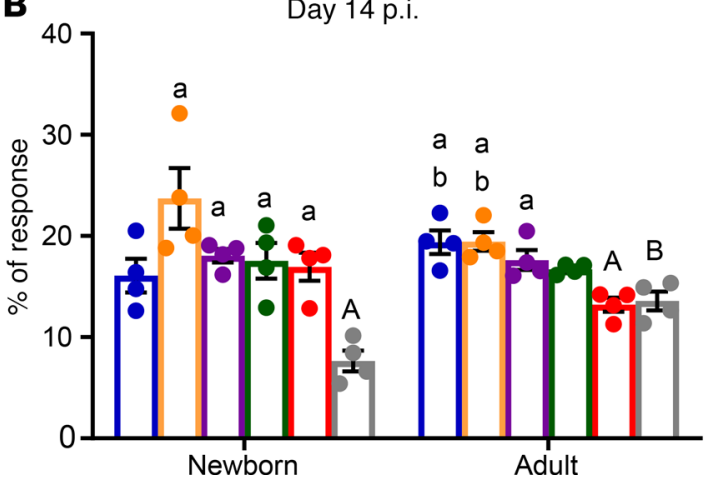

Figure 2. Differences in ID in the early IgM response between newborn and adult AGMs following IAV infection. To measure Abs against each of the identified neutralizing $\mathrm{HA}$ head epitopes, a panel of $\triangle 4 \mathrm{HA}$ constructs were used, each of which had mutations in 4 of the 5 antigenic sites. The name designates the remaining epitope. IgM binding to each construct was assessed at day 8 (A) or day 14 (B) following PR8 infection ( $n=4$ /group). The starting dilution of plasma was 1:10. Following AUC calculation, the percentage of the response based on the WT HA AUC was determined. Significance was determined by 1-way repeated-measures (RM) ANOVA with a Holm-Šídák test for multiple comparisons. Significant differences in binding between epitopes are denoted by lowercase letters corresponding to an index capital letter, e.g., "A" versus "a," where values with the "a" are significantly different (threshold $P<0.05$ ) from those with the "A."

To test this last possibility, we used a recombinant S12 molecule that was produced in HEK293 cells and thus could not have non-HA influenza virus proteins. This analysis showed the Abs bound similarly to both recombinant and virally derived S12 (Figure 5). These data also show that Abs recognizing the recombinant (Figure 5A) or virion-derived (Figure 5B) S12 are the result of infection, as they were absent in plasma from naive animals but significantly increased at day 14 p.i.

Having ruled out the possibility of contaminating proteins in the S12 preparation, we investigated whether Abs present in the plasma from infected animals could recognize the HA stem region. We evaluated IgM, IgG, and IgA Abs at day 14 p.i. using a stabilized California/09 HA stem construct lacking the globular head (33). IgM capable of recognizing the stem construct was significantly induced in adults, and levels were elevated in newborns, though the increase was just below statistical significance, likely due to the variability among the infants (Figure 6A). Both newborn and adult animals generated a robust antistem IgG response (Figure 6B). Stem-specific IgG was also present in the BAL of newborns and adults at day 14 p.i., albeit at low levels (Figure 6C). Although there was a trend toward higher levels of stem-specific $\mathrm{Ab}$ in the respiratory tracts of adults compared with newborns, the data did not reach statistical significance. These data show for the first time to our knowledge that a newborn can mount an $\mathrm{Ab}$ response against the conserved HA stem region following primary infection with IAV. To further define these Abs, we used the well-characterized stem mAb CR9114 (34) as a competitor for binding to stem or HA. CR9114 inhibited IgG binding to stem in a dose-dependent manner (Figure 6D), showing that the Abs generated in the infected animals and CR9114 recognize a similar epitope.

We also evaluated IgA Ab against the HA stem region. Interestingly, in the plasma an IgA stem-specific $\mathrm{Ab}$ response was observed in only 1 adult (Figure 6E), and only 2 of 4 adult animals had detectable IgA Ab against stem in the BAL (Figure 6F). No HA stem-specific IgA was detected in infants. These data suggest IgA $\mathrm{Ab}$ directed against the HA stem is less efficiently generated compared with IgG $\mathrm{Ab}$.

The HA stem-specific Ab response generated by infection is both neutralizing and high avidity. We next measured the avidity of the stem-specific IgG $\mathrm{Ab}$ as an indicator of Ab quality. No difference in avidity between newborns and adults was observed for IgG binding to the stabilized Ca09 headless stem construct (Figure $7 \mathrm{~A})$, suggesting that at this point in the acute response when infection is actively being cleared, newborn $\mathrm{Ab}$ against stem should have similar activity based on avidity. To further examine the quality of these Abs, we assessed the capacity of the elicited stem Abs to neutralize virus using a chimeric virus expressing an $\mathrm{H} 5$ 
A

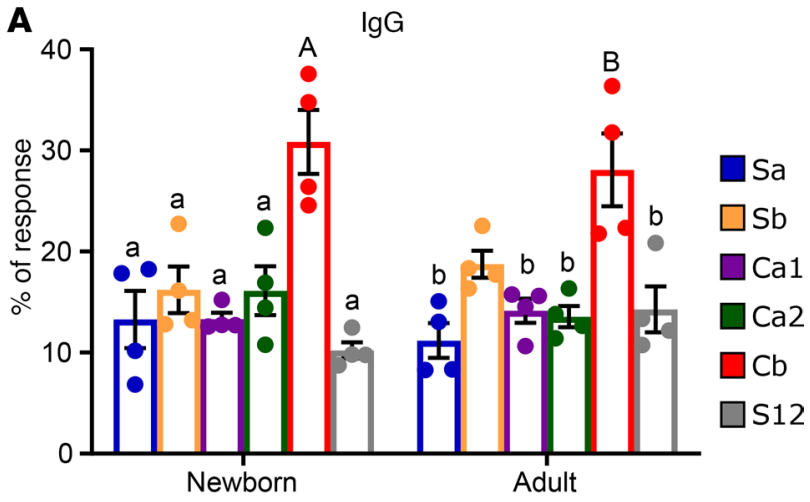

B

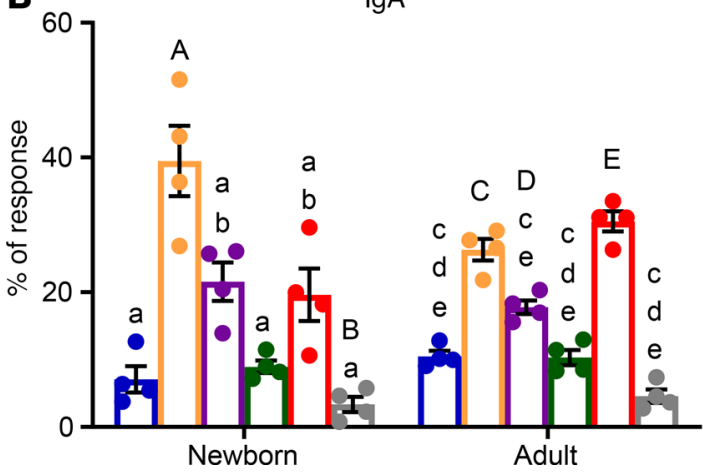

Figure 3. ID hierarchy of HA-specific IgG and IgA $A b$ in newborn and adult AGMs. The proportion of IgC (A) and IgA (B) that binds each of the $\triangle 4 \mathrm{HA}$ molecules relative to WT HA-specific Ab in newborn and adult AGM at day 14 p.i. was determined ( $n=4$ /group). Ab binding to each site was calculated as in Figure 2. The initial plasma dilution was 1:160 for IgG and 1:20 for IgA. Significance was determined by 1-way ANOVA with a Holm-Šídák test for multiple comparisons and is denoted as described in Figure 2. The threshold for significance was $P<0.05$.

head, H1 PR8 stem, and N2 neuraminidase (H5/H1-N2). The substitution of a heterologous globular head and neuraminidase should restrict $\mathrm{Ab}$ binding to the stem, such that any neutralization observed can be attributed to stem-specific Ab. Plasma collected at day 14 p.i. from both age groups was able to neutralize the chimeric H5/H1-N2 virus (Figure 7B), demonstrating that at this time point the stem-specific Abs elicited by infection have comparable neutralizing capacity. These Abs did not affect neuraminidase activity as has been reported for some Abs (35) because neuraminidase activity of the chimeric virus was not inhibited as measured via an enzyme-linked lectin assay (data not shown).

\section{Discussion}

Our understanding of the immune response generated following IAV infection of newborns remains highly limited. Here we have used an outbred NHP model that closely mimics human newborn immune development and function to probe the breadth and specificity of the $\mathrm{Ab}$ response generated following initial exposure to IAV. By using this model, we expand the translatability of these findings to humans and probe the potential for genetic heterogeneity to affect $\mathrm{Ab}$ ID.

We found that newborns and adults establish similar IgG dominance profiles to the 5 main antigenic sites of $\mathrm{H} 1 \mathrm{HA}$, while exhibiting distinct patterns of ID in the IgA and early IgM response. Consistent with studies in adult mice (17), avidity was not associated with dominance. We also show for the first time to our knowledge isotype-dependent divergence in ID, with IgA exhibiting a distinct profile compared with IgG in both newborns and in adults. Finally, we report that by 14 days following primary exposure, newborns have generated robust levels of stem-specific Abs that are similar to those generated by adults in avidity and neutralizing potential.

We were intrigued to find a disparity in the HA-specific IgM Ab present in naive infants versus adults. This was coupled with an early difference in the dominance pattern for IgM between these 2 groups. Given the reduced control of virus in infants at day 2 and day 8 p.i. that we have previously observed, it is tempting to speculate that these early differences affect viral load. Alternatively, it is possible that viral load is driving the divergence in early responses. In mice, natural IgM (nIgM) Abs appear to play an important role in limiting IAV replication (36). These Abs promote viral clearance in part through complement-mediated neutralization $(36,37)$. Because $\mathrm{nIgM}$ production is attributable to B1 cells, our finding may reflect a deficit in the $\mathrm{B} 1$ compartment of newborn primates, consistent with the poor responses to 

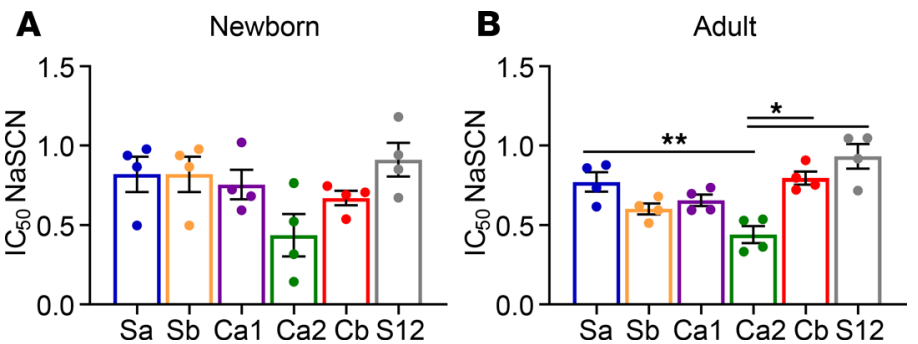

Figure 4. Antigenic site-specific IgG Ab avidity in newborn and adult AGMs. The avidity of IgG binding to each epitope was determined by disruption with NaSCN in plasma collected at day 14 p.i. from newborns (A) and adults (B). The Ab dilution used in the assay for each animal was that which gave $50 \%$ of maximal binding to WT HA to normalize for the total amount of HA-specific $A b$ in the assay. To measure avidity, increasing concentrations of $\mathrm{NaSCN}(0.5 \mathrm{M})$ were added to plates to disrupt binding. Shown is the NaSCN concentration giving a $50 \%$ reduction in optical absorbance compared with the untreated sample. Significance was assessed by RM 1-way ANOVA with Tukey's multiple comparisons; ${ }^{*} P<0.05$, and ${ }^{*} P<0.01$.

T-independent antigens reported in this age group $(38,39)$. An alternative, but not exclusive, possibility is that there is a difference in the initial recruitment and/or activation of B cells because we would expect extrafollicular B2 cells to be a significant contributor to the IgM present in circulation at day 8 (40).

The finding that $\mathrm{Cb}$ dominates the IgG response at day 14 p.i. in both newborns and adults is striking in its similarity to the pattern reported in a mouse model of PR8 infection (17). Cb-specific Abs have been observed to dominate the early $\mathrm{Ab}$ response across a variety of models, preferentially differentiating into extrafollicular plasmablasts rather than adopting a germinal center fate $(41,42)$. This may be the result of a unique aspect of the response to $\mathrm{Cb}$, whether it be the $\mathrm{B}$ cell repertoire as was suggested by Kavaler et al. (43) or an intrinsic property of the antigen. Interestingly, while the majority of $\mathrm{Ab}$ specificities were of similar avidity, this dominant $\mathrm{Cb}$-specific IgG response exhibited decreased avidity in newborns compared with adults. It is possible that this has consequences for clearance, considering the established role of $\mathrm{Cb}$-binding $\mathrm{Abs}$ in inhibiting viral egress following replication (44). In mouse models, the early dominance of $\mathrm{Cb}$ gives way to the emergence of Sb-specific IgG at later times (17). Unfortunately, we were unable to assess time points beyond day 14 in our study because animals were euthanized for examination of lung pathology (9). Analysis of the evolution of ID in newborns and the establishment of ID in the memory B cell population in future studies will provide important complementary insights into immune regulation in this population.

Evaluation of ID within the HA-specific IgA Ab of newborns and adults revealed an unexpected divergence from IgG, as Cb-specific Abs were not dominant among IgA Abs from either age group. Our finding differs from a recent study by Kang et al. evaluating an HIV vaccine in the macaque model (45). Here IgG and IgA had similar patterns of binding to epitopes from the HIV Env protein. This difference may be the result of evaluation at a later time point (week 80 ) following vaccination, reflecting evolution of the $\mathrm{Ab}$ response. Alternatively, distinct attributes of the pathogen or nature of the antigen exposure (i.e., HIV versus IAV or vaccination versus infection) may be responsible for the divergent outcomes.
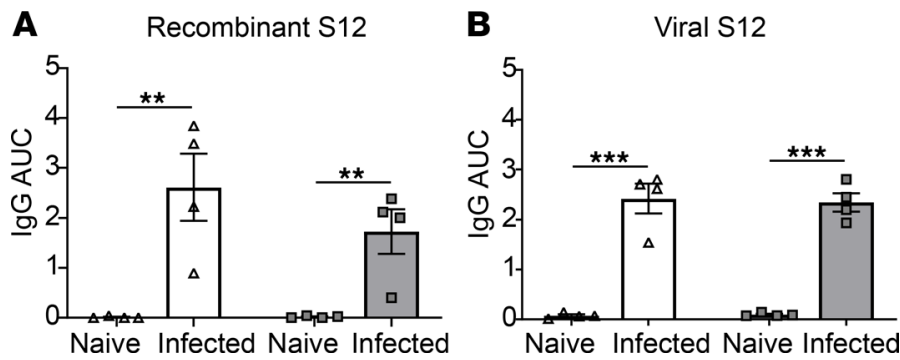

Figure 5. Generation of Ab capable of recognizing S12 in newborn and adult AGMs following IAV infection. (A) Plasma from newborn or adult naive animals or animals infected with PR8 14 days prior was tested for recognition of the recombinant $\mathrm{S} 12$ HA protein. The starting dilution of plasma was 1:80. (B) Plasma from naive and infected animals was also tested for recognition of virally derived S12 HA. Significance was determined by unpaired 1-tailed $t$ test; ${ }^{* *} P<0.01$, and ${ }^{* * *} P<0.001$. 

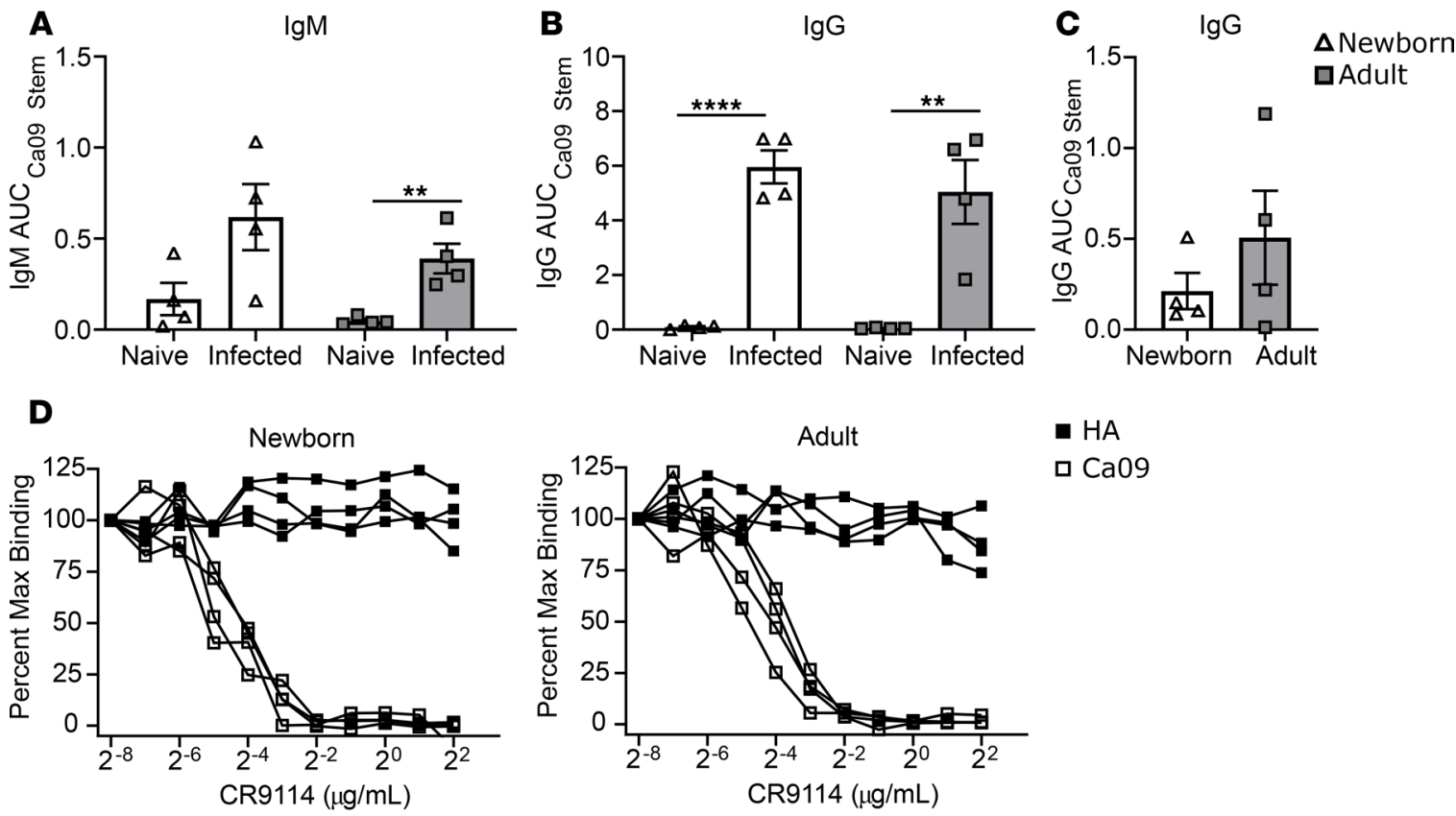

$$
\text { - HA }
$$

口 $\mathrm{Ca09}$
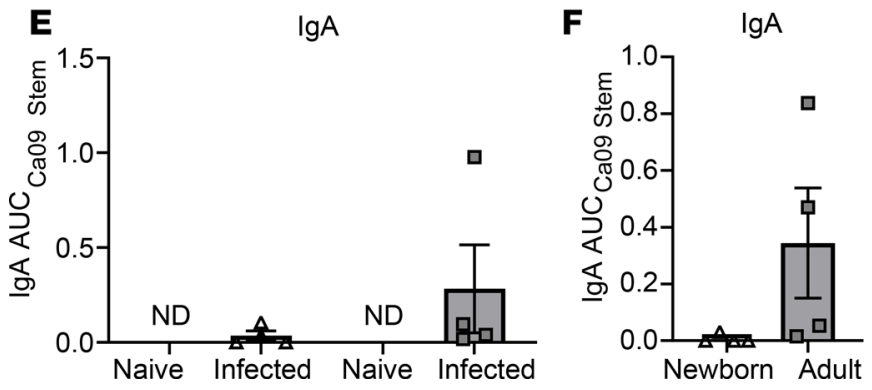

Figure 6. HA stem-specific Ab response in newborn and adult AGMs following IAV infection. IgM (A) and IgC (B) binding to the HA stem region in plasma samples from naive and infected animals ( $n=4$ /group) was quantified by ELISA using a headless California/09 HA stem construct. (C) The presence of stem-specific IgG was also assessed in BAL collected at day 14 p.i. Pre- and postinfection samples for adults are from the same animals. Naive newborn samples are from an age-matched control cohort. (D) The capacity of stem mAb CR9114 to competitively inhibit IgC binding to HA and Ca09 was assessed by ELISA in plasma collected at day 14 p.i. from newborns and adults. Plates were preincubated with serial dilutions of CR9114. Plasma was diluted at 1:80 and added to plates coated with HA or the stem construct as indicated. IgA Ab in the plasma (E) and BAL (F) collected at day 14 p.i. was also evaluated for $\mathrm{Ab}$ binding to the stem region. Plasma IgM and IgA assays were performed starting at a dilution of 1:10 and 1:20, respectively. The starting dilution for plasma IgG was 1:80 because of the high HA-specific Ab titer at day 14 p.i. BAL assays were performed at a starting dilution of 1:5. Significance was determined by unpaired 1-tailed $t$ test; ${ }^{* *} P<0.01$, and ${ }^{* * *} P<0.0001$.

There are a multitude of factors that may drive the IgA response in early life, including alterations in the cytokine milieu, changes in neonatal $\mathrm{T}$ follicular helper cells, and the diminished ability to undergo affinity maturation $(10,13,46,47)$. All of these are potential contributors to ID. Identification of those responsible for these differences awaits further study.

There is now intense focus on the development of approaches that can optimally elicit Abs that recognize the relatively conserved stem region of HA, given the capacity of these Abs to provide broad protection (48). Stem-binding Abs can function by inhibiting viral entry or release (49) as well as Fc receptor-mediated clearance $(50,51)$. HA stem-specific responses are challenging to elicit because this region is poorly immunogenic, especially in the context of current vaccines $(31,32)$. However, HA stem-specific Abs in humans are known to increase with age, presumably because of multiple exposures to heterologous strains of IAV, supporting the ability of these responses to be boosted $(19,31,33,52)$. Given the challenges associated with studying young human infants and the difficulty in knowing the exposure history in the first years of life, there is limited information regarding the capacity for these individuals to generate the highly sought-after stem-specific Abs upon IAV infection. This issue is significant because a lower capacity for HA stem-specific Ab production in newborns would be predicted to put them at a significant disadvantage in the event of infection with a novel pandemic strain (53). 

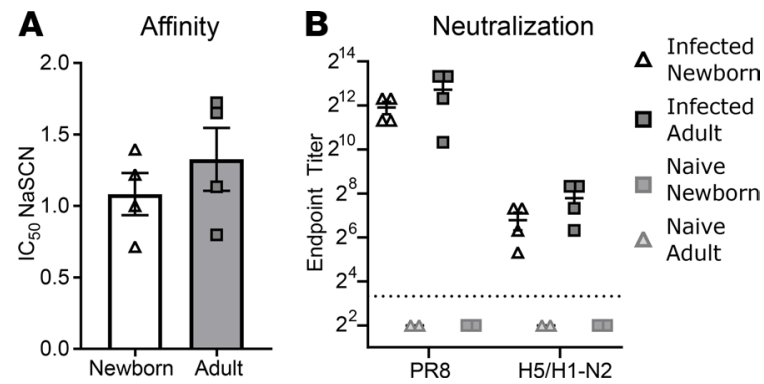

Figure 7. Avidity and neutralizing capacity of stem-binding Ab elicited in newborn and adult AGMs. (A) Avidity of IgG to the California/09 stem construct was calculated as the NaSCN concentration giving a 50\% reduction in optical absorbance compared with the untreated sample. (B) Neutralizing stem-specific Ab present at day 14 p.i. was assessed using a chimeric virus bearing an $\mathrm{H} 5$ head and $\mathrm{N} 2$ neuraminidase to limit detectable neutralization to stem-binding Abs. The limit of detection for the assay is designated by the dashed line; representative naive samples were all below threshold.

We found a robust stem-specific IgG response in the newborns infected with PR8. This is an exciting result and provides rationale for targeting these responses in young infants. In addition, when evaluated at the day 14 p.i. time point, the avidity of $\mathrm{Ab}$ binding to the HA stem in newborns was comparable to $\mathrm{Ab}$ binding to the other HA epitopes and was similar to that in adults. Importantly, these Abs were able to neutralize influenza virus.

Abs directed against the stem region of HA isolated from humans preferentially utilize a set of $\mathrm{V}_{\mathrm{H}}$ regions, including $V_{H} 1-69$ for group $1 \mathrm{HA}$ stem and $V_{H} 1-18$ and $V_{H} 6-1$ for crossgroup stem reactivity (54-58). These Abs are characterized by low to moderate amounts of somatic hypermutation (SHM) (59). Pappas et al. reported that a single mutation could confer high affinity for the stem region (54). The B cell repertoire in newborns versus adults is distinct (60-63). Specifically, neonate B cells have a nearly germline $\mathrm{V}_{\mathrm{H}}$ repertoire (61) and exhibit a reduced range of IgM heavy-chain complementarity-determining region 3 (HCDR3) lengths; i.e., the CDR3 is significantly longer in adult B cells $(62,63)$. The breadth and complexity of the repertoire of $\mathrm{B}$ cells in newborns that can respond to influenza virus are relatively unexplored. However, the limited SHM required by many HA stem-reactive Abs may reduce the barrier to eliciting these Abs in infants and may contribute to the production observed in our study.

It was surprising that circulating IgA Abs against the HA stem were largely absent in both newborn and adult animals. The modest IgA response to stem is particularly noteworthy in light of recent studies demonstrating more potent neutralization by heterosubtypic IgA than IgG that appears to result from intrinsic characteristics of the $\operatorname{IgA}$ constant region $(64,65)$. Further studies to identify the mechanism responsible for the poor generation of stem-specific IgA Ab following infection may facilitate design of vaccine regimens with improved capacity to elicit IgA Ab against stem that may provide increased protection.

In summary, the findings presented here show that establishment of HA-specific Ab ID is dependent on age and differs by isotype. The observation that the dominant epitope recognized by HA-specific IgG at day 14 p.i. is similar in newborns and adults together with the finding that newborns can generate stem-specific Abs suggest the newborn B cell repertoire does not represent a bottleneck for developing HA stemspecific responses. These data are supportive for continued pursuit of a universal influenza vaccine for use in this population. It will be important for future studies to evaluate the later phases of the Ab response in this model as well as the ID pattern within the memory B cell pool. We also note that these patterns could differ following exposure to nonreplicating virus as is the case during vaccination. This will be an important area for future study. Finally, the difference identified in the early IgM response in newborns versus adults emphasizes the need for a generally increased understanding of the early Ab response in newborns. Together, these findings provide new insights into the dynamics and quality of the Ab response that is elicited in the neonatal immune system following infection with influenza virus.

\section{Methods}

Study design. The main objective of this study was to compare differences in Ab ID to IAV HA between newborn and adult NHPs. Newborn animals were chosen based on animals born in the time frame that would allow concurrent entry into the study. Plasma and respiratory samples were collected from the newborns (3 male, 1 female) and adults (all female) following infection with IAV. Samples were analyzed 
using engineered HA probes to quantify Ab binding to specific head and stem epitopes by ELISA. Sample sizes are denoted in the text and in figure legends. There were no data points designated as outliers or excluded from analysis. Day 14 p.i. was prospectively selected as the data endpoint for all animals in the interest of collecting tissue and BAL via necropsy at a time point when adaptive immune responses in the lung were expected to be robust.

Animals and infection. Adult and newborn AGMs used in this study were housed at the Wake Forest School of Medicine (WFSM) Vervet Research Colony. The WFSM animal care and use protocol adhered to the US Animal Welfare Act and Animal Welfare Regulations. Animals were infected as described previously (9). In brief, newborns (6-10 days of age) received $1 \times 10^{9}$ (3 infants) or $1 \times 10^{8}$ (1 infant) 50\% egg infectious dose $\left(\mathrm{EID}_{50}\right)$ PR8 divided equally between intranasal and intratracheal routes. The combined infection approach was selected based on prior NHP IAV challenge studies $(66,67)$. There were no differences in the findings between the infants that received the lower dose versus those receiving the higher dose, and thus these infants were grouped for the analyses presented. Adults (6-9 years of age) were infected with $5 \times 10^{9}$ $\mathrm{EID}_{50}$ in the same manner. Adult animals received more virus to adjust for differences in body size. Blood was collected by venipuncture into sodium heparin tubes at day 8 and day 14 p.i., from which plasma was obtained. BAL using $5 \mathrm{~mL}$ and $25 \mathrm{~mL}$ PBS for infants and adults, respectively, was performed at necropsy on day 14 p.i. Samples were centrifuged to remove cells, and BSA was added to a concentration of $0.5 \%$.

HA ELISAs. ELISAs were performed as previously described in Angeletti et al. (17) with modifications for the NHP model. In brief, plates were coated overnight with 5 ng of purified HA probes (17) or a headless California/2009 HA stem construct (33). Plates were blocked with $50 \mu \mathrm{L}$ of casein blocking buffer plus $2 \%$ goat serum for 1 hour, after which they were washed 5 times with PBS plus $0.01 \%$ Tween-20 (PBST). Plates were incubated for 3 hours with $25 \mu \mathrm{L}$ plasma serially diluted in blocking buffer by 2 -fold. Starting dilutions were as stated for each assay. Plates were then washed 5 times in PBST and subsequently incubated with $25 \mu \mathrm{L}$ HRP-conjugated goat anti-NHP IgG (43R-IG020HRP, Fitzgerald), IgM (LS-C86516, LifeSpan Bioscience), or biotinylated goat anti-NHP IgA (MCA2553B, AbD Serotec). For IgA detection, plates underwent an additional wash and incubation with $25 \mu \mathrm{L}$ streptavidin-conjugated $\mathrm{HRP} \mathrm{Ab}$ (33109, Mabtech). All plates were washed 5 times with PBST and developed for 5 minutes using Ultra TMB (Thermo Fisher Scientific), after which the reaction was stopped with $0.1 \mathrm{~N} \mathrm{H}_{2} \mathrm{SO}_{4}$. Binding was expressed as AUC calculated using GraphPad Prism. Calculated AUC was divided by the PR8 HA AUC value to normalize for differences in absolute HA titer among the animals. Plasma binding to uncoated wells was subtracted for each animal, and limit of detection for the AUC calculation was defined as a value that exceeded the average $\mathrm{OD}_{450}$ of the uncoated wells plus 2 standard deviations.

Avidity. Avidity assays were performed as the ELISAs were with the addition of an NaSCN dissociation step following sample incubation. The plasma dilution used was selected for each animal based on the dilution that yielded $50 \%$ of the max $\mathrm{OD}_{450}$ in the ELISA binding curve. Following incubation with $25 \mu \mathrm{L}$ plasma, 2-fold dilutions of NaSCN starting at $5 \mathrm{M}$ were added to the plate for 15 minutes. Plates were then washed 5 times with PBST and incubated with HRP-conjugated goat anti-IgG for 1 hour and developed as in the ELISA protocol. The $\mathrm{IC}_{50}$ was calculated using GraphPad Prism software.

Ab competition. As with the ELISAs, plates were coated with $5 \mathrm{ng}$ of HA or $\mathrm{Ca} 09$ stem probe overnight and blocked for 1 hour with casein blocking buffer plus 2\% goat serum. Plates were then washed with PBST and preincubated with CR9114 mAb in 2-fold serial dilutions for 1 hour. Ab was flicked off and $25 \mu \mathrm{L}$ plasma at a 1:80 dilution in casein blocking buffer was added to each well. After 3 washes with PBST, a human adsorbed HRP-conjugated goat anti-NHP IgG detection Ab (A140-202P, Bethyl Laboratories) was added for 1 hour. Plates were then washed again in PBST and developed for 5 minutes with Ultra TMB substrate and stopped with $0.1 \mathrm{~N} \mathrm{H}_{2} \mathrm{SO}_{4}$ as in the ELISA. The $\mathrm{OD}_{450}$ was used to calculate the percentage of signal seen in the sample with no CR9114 competition.

Microneutralization. Microneutralization assays were performed on Madin-Darby Canine Kidney (MDCK; ATCC) cells as previously described (17). Briefly, 2-fold diluted plasma samples from naive and infected NHPs were incubated with $10050 \%$ tissue culture infectious dose $\left(\mathrm{TCID}_{50}\right)$ of either PR8 or a viral construct with a chimeric HA consisting of an H5/Vietnam/04 head and an H1/PR8 stem as well as an N2 neuraminidase from A/Udorn/72/H3N2 in TCID $_{50}$ medium (DMEM + GlutaMAX + 1\% HEPES + $1 \mu \mathrm{g} / \mathrm{mL}$ L-1-tosylamide-2-phenylethyl chloromethyl ketone-treated trypsin). Samples were incubated for 1 hour at room temperature and added in quadruplicate to confluent MDCK cells, then maintained at $37^{\circ} \mathrm{C}$. Cytopathic effects were assessed after 3 days by microscopy and confirmed by crystal violet staining following methanol fixation. 
Statistics. Statistical significance for pairwise comparisons (e.g., newborns vs. adults; threshold $P<0.05$ ) was determined using 1-tailed unpaired $t$ tests. Statistical comparisons of Ab binding to HA epitopes (Figures 2 and 3) were assessed by 1-way ANOVA with a Holm-Šídák test for multiple comparisons. Differences in Ab avidity (Figure 4) were assessed for significance using an RM 1-way ANOVA with the Greenhouse-Geisser correction and Tukey's multiple-comparisons test. Data were assessed for potential sex-dependent differences in $\mathrm{Ab}$ response. No clear trends were apparent.

Study approval. All animal protocols were approved by the Institutional Animal Care and Use Committee at WFSM.

\section{Author contributions}

EC designed and performed experiments, analyzed data, and wrote the paper. BCH performed NHP infections and sampling. DA and MK generated critical reagents for the study. MJJ facilitated the choice of animals and infection of NHPs as well as managed the NHP colony and oversaw all animal procedures. MAAM designed experiments and wrote the paper. JY and BSG provided critical insight into experimental design. All authors contributed to editing of the final manuscript.

\section{Acknowledgments}

We thank the Wake Forest Animal Resources Program and the veterinary and technical staff of the Vervet Research Colony for care of animals and assistance with animal procedures. These studies were supported by NIH R01 AI098339 (to MAAM) and a grant from the Wake Forest University Clinical and Translational Science Institute (UL1 TR001420). The Vervet Research Colony is supported in part by NIH P40 OD010965 (to MJJ). JY is supported by Division of Intramural Research funds and BSG by Vaccine Research Center funds from the National Institute of Allergy and Infectious Diseases.

Address correspondence to: Martha A. Alexander-Miller, Wake Forest School of Medicine, Biotech Place Room 2E-018, 575 North Patterson Avenue, Winston-Salem, North Carolina 27101, USA. Phone: 336.716.5936; Email: Martha.Alexander-Miller@wakehealth.edu.

1. Basha S, Surendran N, Pichichero M. Immune responses in neonates. Expert Rev Clin Immunol. 2014;10(9):1171-1184.

2. Elahi S, et al. Immunosuppressive CD71+ erythroid cells compromise neonatal host defence against infection. Nature. 2013;504(7478):158-162.

3. Poehling KA, et al. The underrecognized burden of influenza in young children. N Engl J Med. 2006;355(1):31-40

4. Munoz FM. Influenza virus infection in infancy and early childhood. Paediatr Respir Rev. 2003;4(2):99-104.

5. Groothuis JR, Levin MJ, Rabalais GP, Meiklejohn G, Lauer BA. Immunization of high-risk infants younger than 18 months of age with split-product influenza vaccine. Pediatrics. 1991;87(6):823-828.

6. Macdonald N, Bortolussi R. Protecting young babies from influenza. Paediatr Child Health. 2009;14(9):612-617.

7. Siegrist CA. The challenges of vaccine responses in early life: selected examples. J Comp Pathol. 2007;137(suppl 1):S4-S9.

8. Alexander-Miller MA. Vaccines against respiratory viral pathogens for use in neonates: opportunities and challenges. J Immunol. 2014;193(11):5363-5369.

9. Holbrook BC, et al. Nonhuman primate infants have an impaired respiratory but not systemic IgG antibody response following influenza virus infection. Virology. 2015;476:124-133.

10. Schatorjé EJ, Driessen GJ, van Hout RW, van der Burg M, de Vries E. Levels of somatic hypermutations in B cell receptors increase during childhood. Clin Exp Immunol. 2014;178(2):394-398.

11. Muthukkumar S, Goldstein J, Stein KE. The ability of B cells and dendritic cells to present antigen increases during ontogeny. J Immunol. 2000;165(9):4803-4813.

12. Glaesener S, et al. Decreased production of class-switched antibodies in neonatal B cells is associated with increased expression of miR-181b. PLoS One. 2018;13(2):e0192230.

13. Girschick HJ, Lipsky PE. The kappa gene repertoire of human neonatal B cells. Mol Immunol. 2002;38(15):1113-1127.

14. Debock I, et al. Neonatal follicular Th cell responses are impaired and modulated by IL-4. J Immunol. 2013;191(3):1231-1239.

15. Caton AJ, Brownlee GG, Yewdell JW, Gerhard W. The antigenic structure of the influenza virus A/PR/8/34 hemagglutinin (H1 subtype). Cell. 1982;31(2 pt 1):417-427.

16. Peng B, et al. Comparison of the protective efficacy of neutralizing epitopes of 2009 pandemic H1N1 influenza hemagglutinin. Front Immunol. 2017;8:1070.

17. Angeletti D, et al. Defining B cell immunodominance to viruses. Nat Immunol. 2017;18(4):456-463.

18. Yewdell JW. Confronting complexity: real-world immunodominance in antiviral CD8+ T cell responses. Immunity. 2006;25(4):533-543.

19. Andrews SF, et al. Immune history profoundly affects broadly protective B cell responses to influenza. Sci Transl Med. 2015;7(316):316ra192.

20. He W, et al. Epitope specificity plays a critical role in regulating antibody-dependent cell-mediated cytotoxicity against influenza 
A virus. Proc Natl Acad Sci U S A. 2016;113(42):11931-11936.

21. Wrammert J, et al. Broadly cross-reactive antibodies dominate the human B cell response against 2009 pandemic H1N1 influenza virus infection. J Exp Med. 2011;208(1):181-193.

22. Kosik I, Yewdell JW. Influenza A virus hemagglutinin specific antibodies interfere with virion neuraminidase activity via two distinct mechanisms. Virology. 2017;500:178-183.

23. Cobey S, Hensley SE. Immune history and influenza virus susceptibility. Curr Opin Virol. 2017;22:105-111.

24. Davis AS, Taubenberger JK, Bray M. The use of nonhuman primates in research on seasonal, pandemic and avian influenza, 1893-2014. Antiviral Res. 2015;117:75-98.

25. Altman MO, Bennink JR, Yewdell JW, Herrin BR. Lamprey VLRB response to influenza virus supports universal rules of immunogenicity and antigenicity. Elife. 2015;4.

26. Das SR, et al. Defining influenza A virus hemagglutinin antigenic drift by sequential monoclonal antibody selection. Cell Host Microbe. 2013;13(3):314-323.

27. Klasse PJ. How to assess the binding strength of antibodies elicited by vaccination against HIV and other viruses. Expert Rev Vaccines. 2016;15(3):295-311.

28. Khurana S, et al. MF59 adjuvant enhances diversity and affinity of antibody-mediated immune response to pandemic influenza vaccines. Sci Transl Med. 2011;3(85):85ra48

29. Krammer F, Palese P. Influenza virus hemagglutinin stalk-based antibodies and vaccines. Curr Opin Virol. 2013;3(5):521-530.

30. Zhu W, Wang C, Wang BZ. From variation of influenza viral proteins to vaccine development. Int J Mol Sci. 2017;18(7):E1554.

31. Nachbagauer R, Choi A, Izikson R, Cox MM, Palese P, Krammer F. Age dependence and isotype specificity of influenza virus hemagglutinin stalk-reactive antibodies in humans. mBio. 2016;7(1):e01996-e01915.

32. Tan HX, et al. Subdominance and poor intrinsic immunogenicity limit humoral immunity targeting influenza HA stem. $J$ Clin Invest. 2019;129(2):850-862.

33. Yassine HM, et al. Use of hemagglutinin stem probes demonstrate prevalence of broadly reactive group 1 influenza antibodies in human sera. Sci Rep. 2018;8(1):8628.

34. Dreyfus C, et al. Highly conserved protective epitopes on influenza B viruses. Science. 2012;337(6100):1343-1348.

35. Kosik I, et al. Neuraminidase inhibition contributes to influenza A virus neutralization by anti-hemagglutinin stem antibodies J Exp Med. 2019;216(2):304-316.

36. Baumgarth N, Herman OC, Jager GC, Brown LE, Herzenberg LA, Chen J. B-1 and B-2 cell-derived immunoglobulin M antibodies are nonredundant components of the protective response to influenza virus infection. J Exp Med. 2000;192(2):271-280.

37. Jayasekera JP, Moseman EA, Carroll MC. Natural antibody and complement mediate neutralization of influenza virus in the absence of prior immunity. J Virol. 2007;81(7):3487-3494.

38. Rijkers GT, Sanders EA, Breukels MA, Zegers BJ. Infant B cell responses to polysaccharide determinants. Vaccine. 1998;16(14-15):1396-1400.

39. Baker PJ, Stashak PW, Amsbaugh DF, Prescott B. Characterization of the antibody response to type 3 pneumococcal polysaccharide at the cellular level. I. Dose-response studies and the effect of prior immunization on the magnitude of the antibody response. Immunology. 1971;20(4):469-480.

40. Lam JH, Baumgarth N. The multifaceted B cell response to influenza virus. J Immunol. 2019;202(2):351-359.

41. Kavaler J, Caton AJ, Staudt LM, Gerhard W. A B cell population that dominates the primary response to influenza virus hemagglutinin does not participate in the memory response. Eur J Immunol. 1991;21(11):2687-2695.

42. Rothaeusler K, Baumgarth N. B-cell fate decisions following influenza virus infection. Eur J Immunol. 2010;40(2):366-377.

43. Kavaler J, Caton AJ, Staudt LM, Schwartz D, Gerhard W. A set of closely related antibodies dominates the primary antibody response to the antigenic site $\mathrm{CB}$ of the A/PR/8/34 influenza virus hemagglutinin. J Immunol. 1990;145(7):2312-2321.

44. Brandenburg B, et al. Mechanisms of hemagglutinin targeted influenza virus neutralization. PLoS One. 2013;8(12):e80034

45. Kang ZH, et al. Similar epitope specificities of IgG and IgA antibodies elicited by Ad26 vector prime, Env protein boost immunizations in rhesus monkeys. J Virol. 2018;92(15):e00537-18.

46. Tangye SG, Ferguson A, Avery DT, Ma CS, Hodgkin PD. Isotype switching by human B cells is division-associated and regulated by cytokines. J Immunol. 2002;169(8):4298-4306.

47. Thornburg NJ, Shepherd B, Crowe JE. Transforming growth factor beta is a major regulator of human neonatal immune responses following respiratory syncytial virus infection. J Virol. 2010;84(24):12895-12902.

48. Krammer F, García-Sastre A, Palese P. Is it possible to develop a "universal" influenza virus vaccine? Potential target antigens and critical aspects for a universal influenza vaccine. Cold Spring Harb Perspect Biol. 2018;10(7):a028845.

49. Boudreau CM, Alter G. Extra-neutralizing FcR-mediated antibody functions for a universal influenza vaccine. Front Immunol. 2019;10:440.

50. DiLillo DJ, Palese P, Wilson PC, Ravetch JV. Broadly neutralizing anti-influenza antibodies require Fc receptor engagement for in vivo protection. J Clin Invest. 2016;126(2):605-610.

51. Smith SA, et al. VH1-69 utilizing antibodies are capable of mediating non-neutralizing Fc-mediated effector functions against the transmitted/founder gp120. Front Immunol. 2018;9:3163.

52. Miller MS, et al. Neutralizing antibodies against previously encountered influenza virus strains increase over time: a longitudinal analysis. Sci Transl Med. 2013;5(198):198ra107.

53. Ruf BR, Knuf M. The burden of seasonal and pandemic influenza in infants and children. Eur J Pediatr. 2014;173(3):265-276.

54. Pappas L, et al. Rapid development of broadly influenza neutralizing antibodies through redundant mutations. Nature. 2014;516(7531):418-422.

55. Joyce MG, et al. Vaccine-induced antibodies that neutralize group 1 and group 2 influenza A viruses. Cell. 2016;166(3):609-623

56. Wheatley AK, et al. H5N1 Vaccine-elicited memory B cells are genetically constrained by the IGHV locus in the recognition of a neutralizing epitope in the hemagglutinin stem. J Immunol. 2015;195(2):602-610.

57. Sui J, et al. Structural and functional bases for broad-spectrum neutralization of avian and human influenza A viruses. Nat Struct Mol Biol. 2009;16(3):265-273.

58. Avnir Y, et al. Molecular signatures of hemagglutinin stem-directed heterosubtypic human neutralizing antibodies against influenza 
A viruses. PLoS Pathog. 2014;10(5):e1004103.

59. Chen F, Tzarum N, Wilson IA, Law M. $\mathrm{V}_{\mathrm{H}} 1-69$ antiviral broadly neutralizing antibodies: genetics, structures, and relevance to rational vaccine design. Curr Opin Virol. 2019;34:149-159.

60. Williams JV, Weitkamp JH, Blum DL, LaFleur BJ, Crowe JE. The human neonatal B cell response to respiratory syncytial virus uses a biased antibody variable gene repertoire that lacks somatic mutations. Mol Immunol. 2009;47(2-3):407-414.

61. Sun J, Butler JE. Molecular characterization of VDJ transcripts from a newborn piglet. Immunology. 1996;88(3):331-339.

62. Hong B, et al. In-depth analysis of human neonatal and adult IgM antibody repertoires. Front Immunol. 2018;9:128

63. Shiokawa S, et al. IgM heavy chain complementarity-determining region 3 diversity is constrained by genetic and somatic mechanisms until two months after birth. J Immunol. 1999;162(10):6060-6070.

64. He W, Mullarkey CE, Duty JA, Moran TM, Palese P, Miller MS. Broadly neutralizing anti-influenza virus antibodies: enhancement of neutralizing potency in polyclonal mixtures and IgA backbones. J Virol. 2015;89(7):3610-3618.

65. Maurer MA, et al. Glycosylation of human IgA directly inhibits influenza A and other sialic-acid-binding viruses. Cell Rep 2018;23(1):90-99.

66. Go JT, et al. 2009 pandemic H1N1 influenza virus elicits similar clinical course but differential host transcriptional response in mouse, macaque, and swine infection models. BMC Genomics. 2012;13:627.

67. Matsuoka Y, et al. African green monkeys recapitulate the clinical experience with replication of live attenuated pandemic influenza virus vaccine candidates. J Virol. 2014;88(14):8139-8152. 